\title{
Continuously varying binocular disparity: An exploratory study
}

\author{
W. D. GROMAN ${ }^{1}$ AND R. W. WORSHAM \\ VIRGINIA COMMONWEALTH UNIVERSITY
}

It was hypothesized that viewing an object under conditions of continually varying binocular disparity would result in the perception of a "real" object that would continually change its shape, yet retain its threedimensionality. It was further hypothesized that observed changes would be systematically sequential and repetitive, reflecting systematic and repetitive changes in the stimulus conditions. Two Ss were used. They were shown 12 objects (one at a time) by means of the Groman Continuous Binocular Disparator. An amalysis was made from taped verbal reports. The hypotheses were strongly supported. Other phenomena were noted and described. The observations were categorized on the basis of three types of breakdown of veridicality. Differences between the frequency of breakdown of veridicality in the three categories were found and discussed in terms of a functional theory of veridical perception.

This study deals with the phenomena observed under conditions of continuously changing binocular disparity. It was Wheatstone's discovery that a slight difference in two images, one presented to each eye, could arouse an impression of depth, and he invented the stereoscope to demonstrate this fact (Osgood, 1953). With the stereoscope and its derivatives it is possible to determine various aspects and certain limits of binocular disparity; for example, testing where binocular fusion breaks down and diplopic images are seen. However, stereoscopic studies have generally used static conditions for presenting stimulus material, and the stimulus objects are represented by two-dimensional displays. To alter disparity, for example, would necessitate presentation of a different pair of stimulus slides.

For studying all aspects of binocular vision (static and dynamic) the Continuous Binocular Disparator (Groman, 1964) is a more versatile apparatus. Its main advantages are that it can use two-dimensional or three-dimensional stimulus objects; it can present a continuous range of static views; and it can present continuously changing dynamic disparate views. One can, for example, produce optically the conditions that would occur if the two eyes could move in opposite directions around a stationary object, while main taining normal accomodation and convergence for the $\mathrm{S}$. This is accomplished by exposing to each eye separately, through a prism stereoscope, one of two identical stimulus objects that are fused and seen as one. Rotation of the stimulus objects around their central vertical axes in opposite directions then produces continuous cycles of increasing and decreasing disparate views of the objects. The amount of disparity produced depends on the form and symmetry of the stimulus objects. Two identical two-dimensional squares, for example, will result in a range of angular disparity from 0 to 90 deg, while two identical dolls result in a range from 0 to $180 \mathrm{deg}$ of disparity.

The perceptual phenomena resulting from these particular novel viewing conditions have not been studied previously. One purpose of this study, therefore, was to explore the phenomena and attempt to describe and, if possible, classify them. Only very general hypotheses were considered. It was predicted that viewing two identical objects, one seen by each eye, in a situation of continuously varying binocular disparity, would yield a perception of an apparently single object that would change
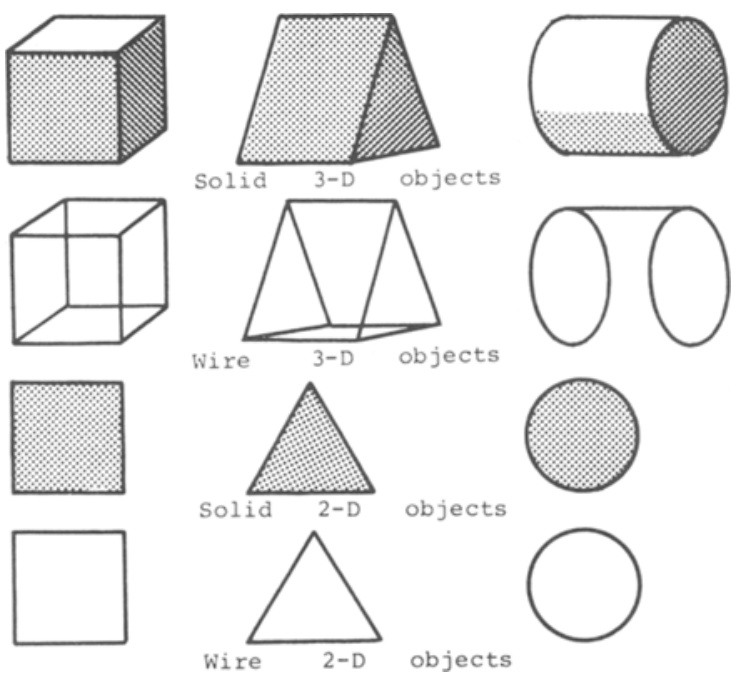

Fig. 1. The 12 stimulus objects.

continuously in form. It was expected that this resultant unified percept would differ markedly from the percept that would result from a simple superimposition of the two optical arrays (Gibson, 1950). It was further hypothesized that the repetitive systematic changes in disparity that constituted the stimulus conditions would be reflected in a systematic repetition of the phenomena reported by the $\mathrm{Ss}$.

\section{Subjects}

\section{METHOD}

The authors served as the two Ss used. One was experienced in visual observation, and the other was relatively inexperienced in observations and descriptions of this type. Both Ss were aware of the nature of the apparatus, and that there were, in each viewing period, actually two identical stimulus objects, one presented to each eye.

\section{Apparatus}

The Groman Continuous Binocular Disparator was used. It was driven by an electrical reduction motor (Bodine Electric Company, Speed Reducer Motor, NSE-12RH), which was connected to a rheostat (Superior Electric Company, Powerstat, $116 \mathrm{U} \mathrm{PH} 1$ ); thus the rotation speed of the stimulus carriers could be varied. The power source was channeled through a Ray theon Line Voltage Regulator (catalog No. RVA 60) in order to control electrical variation. The lighting consisted of two 18-in. flourescent lamps placed perpendicular to the barrier at a height 9 in. above the stimulus carriers and each at a distance of 15 in. from the center of the apparatus. A white matte-finish cardboard was used as the background field, placed 6 in. behind the stimulus objects. The Ss could manipulate a switch to start or stop rotation of the stimulus carriers. Tape recordings were made of the $\mathrm{Ss}^{\text {' descriptions. }}$ 
Table 1

$A=$ The Phenomena Observed with Each Stimulus Object Pair. B = Frequency of Loss of Object Unity, Brightness Unity, and Form Constancy.

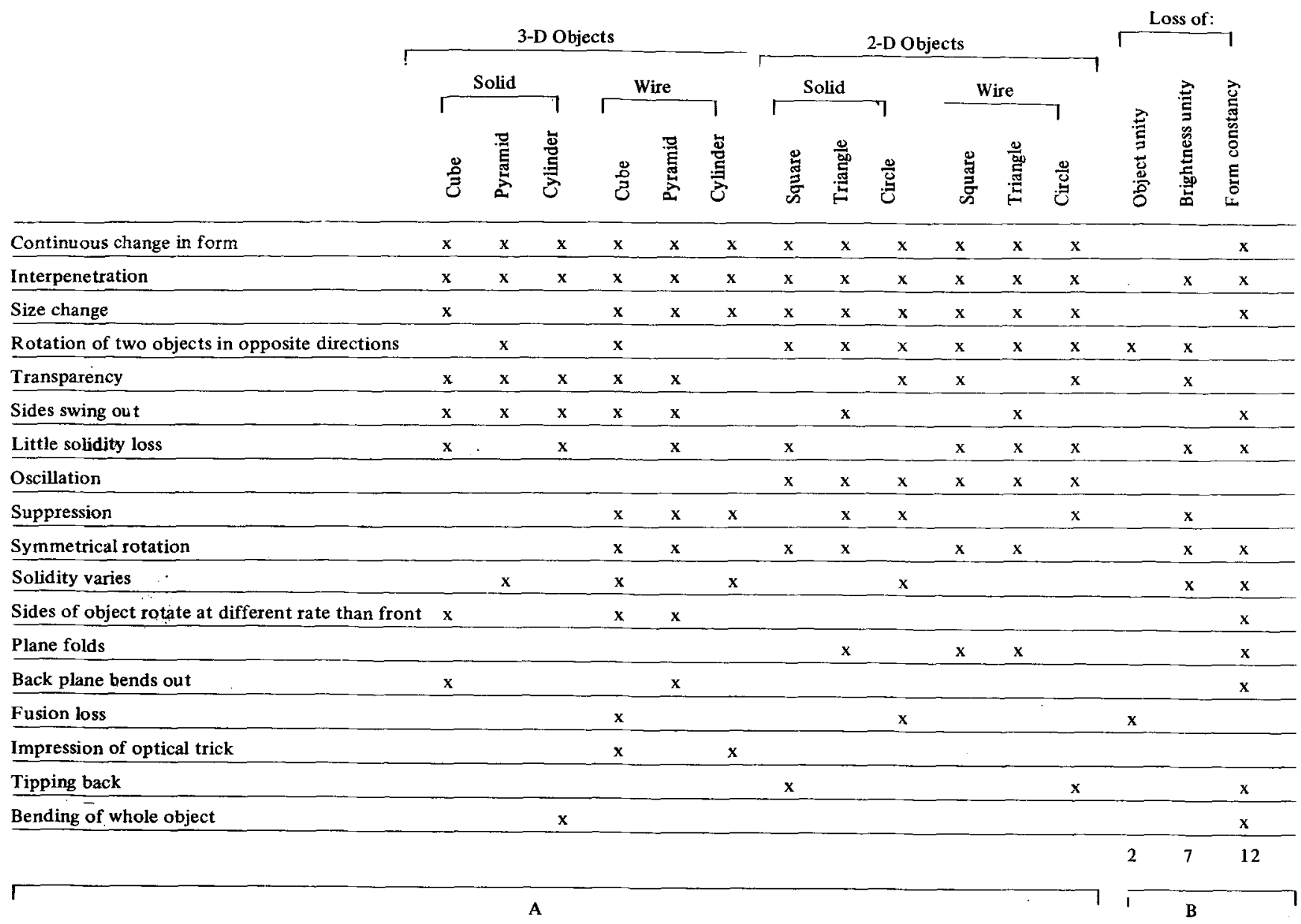

The 12 duplicate stimulus objects consisted of every possible combination of conditions described by a 3 by 2 by 2 matrix. There were three basic geometric shapes: the triangle, the circle, and the square. There were two-dimensional and threedimensional stimulus objects. An object was either solid or wire-outlined. All external dimensions of the objects were $1 \frac{1}{2}$ in. (circle diameters were also $1 \frac{1}{2}$ in.). The objects are diagrammed in Fig. 1. In order to minimize the depth cue of intensity difference at the boundary of the objects (Treisman, 1962), the stimulus objects were spray-painted flat gray.

\section{Procedure}

For each of the 12 presentations, one of the two identical stimulus objects was placed on each stimulus carrier. The S's first view of them, when seated with his head in the chin rest, resulted in the perception of a stationary single geometric object, normal in every respect ${ }^{2}$ and at an apparent distance of about 18 in. $S$ described the initial normal view, then rotation of the stimulus carriers was started. Free viewing was used since it had been found previously that viewing a standard fixation point under these conditions made it very difficult to attend to the changing phenomena well enough to describe them adequately. The complete descriptions were tape-recorded. Each of the 12 presentations was viewed from 7 to $10 \mathrm{~min}$, the time being determined by the length of the S's descriptions. Viewing order of the stimulus objects followed no systematic pattern. Rate of rotation of the stimulus carriers was always $3 \mathrm{rpm}$. The Ss observed four stimulus objects in each of three sessions with a 1-week interval between sessions. A listing and frequency count of the phenomena was made from the tapes (see Table 1).

\section{RESULTS}

Most of the phenomena observed were complex and unlike anything occurring in normal experience, since the conditions of binocular disparity lie far outside the range of possible normal stimulation. A written description does not do justice to the observed phenomena, and one must observe for oneself to apprehend truly the effects produced by the apparatus.

To conserve space, only the more general phenomena will be presented, along with a representative description. The more general phenomena occurring with the respective objects are presented in Table 1 (left). The last three columns of Table 1 (right) are an attempt to classify the mechanisms of veridicality that fail when the respective pheriomena occur. Each phenomenon occurring for a particular object was not necessarily present during the entire viewing period; however, the general rule was that a given phenomenon reoccurred at the same point as part of a cyclic sequence of changes that took place during a given viewing period.

Some of the phenomena listed in the table are self-explanatory. 


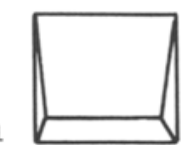

a

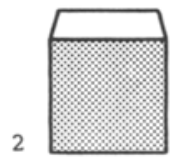

a

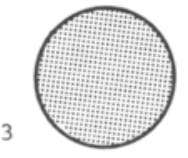

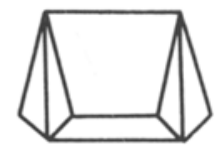

b

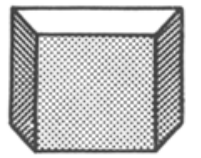

b

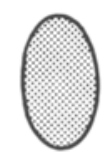

b

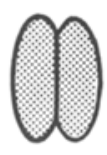

c

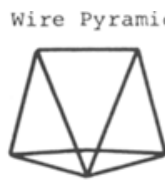

c

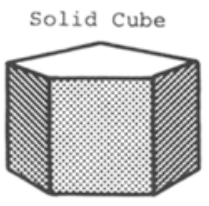

c

Solid Circle

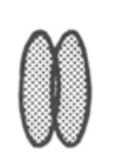

d

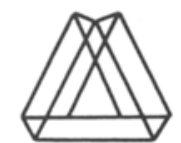

d

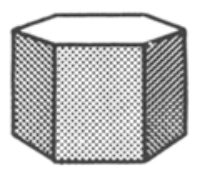

d

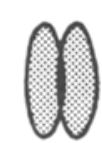

d'

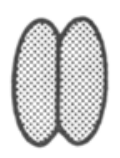

$c^{\prime}$

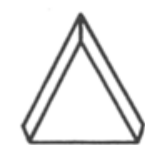

e

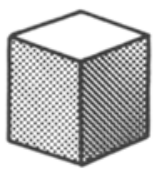

e

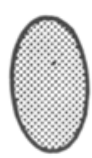

$b^{\prime}$

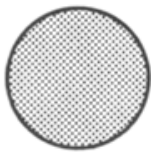

$a^{\prime}$
Fig. 2. Observed sequence changes for three stimulus objects.
Others need further clarification due to the uniqueness of the percepts. The following describes briefly the latter:

(1) Interpenetration-two identical objects appear to occupy the same physical space.

(2) Rotation in opposite directions-two identical objects appear to rotate about the same vertical axis in opposite directions, but some planes of the objects appear to move at different angular rates.

(3) Symmetrical rotation--same as (2), but all parts of each object appear to rotate at the same angular rate.

(4) Solidity-the impression of a real object is maintained, al though the form is observed to change.

(5) Plane folds - a vertical plane seems to fold along a vertical line.

(6) Back plane bends out-same as (5), but refers only to the rear plane of a 3-D object (see Drawing 2c).

(7) Fusion loss-appearance of two objects, due to occasional loss of convergence (see Drawing 3d).

(8) Tipping back - object seems to tip backward slightly, top away from the observer.

(9) Bending of the whole object-analogous to the way a frankfurter bends in a frying pan.

One interesting finding is that the resultant overall perception of an object was dependent upon where one fixated. A different dynamic effect was obtained by changing the point of fixation. Rates of movement of parts of the apparently single stimulus object appeared to be slower at the fixation point and faster at the periphery.

When rotation began, the normally appearing object immediately seemed to distort itself, yet retained its threedimensional solidity. As the disparity increased, it reached a certain critical range, and usually the solidity markedly decreased. When this occurred, two identical objects appeared to be occupying the same physical space, interpenetrating one another, while rotating in opposite directions. During this phase the probability of the suppression of one eye was maximum. The occurrence of peripheral transparencies was most frequent during this phase. As rotation continued, and the disparity decreased toward the normal, the percept became one of a distorted solid object changing its shape to become normal again. When normality was achieved the cycle started anew. The loss of fusion throughout the wide range of unusual conditions was surprisingly small, probably occurring no more than 5 to $10 \%$ of the time.
The complexity of the phenomena observed is related to the nature of the stimulus objects. Highest in complexity were the three-dimensional wire figures. Second in complexity were the three-dimensional solid figures. Least in complexity were the two-dimensional figures. Figure 2 diagrams the most typical apparent changes occurring in three of the stimulus objects.

\section{DISCUSSION}

Both hypotheses were strongly supported. Continuous change in form was observed in all 12 stimulus objects throughout $90-95 \%$ of the viewing time. Also, as expected, changes were, without exception, systematically sequential and repetitive, following the cyclic repetition of the stimulus conditions.

Our results appear to pose some problems for customary ways of accounting for binocular fusion. For example, different parts of our display fall within Panum's area, and we would expect fusion at different times on that account. Panum's area refers to corresponding areas of the two retinae, demonstrating a less rigorous requirement for fusion than point-to-point correspondence. Its magnitude is determined in minutes of arc. In our display conditions, however, disparity occurs (a) in abnormally large numbers of degrees, and (b) some surfaces of the apparently single object perceived are seen by only one eye. The perception of a single object under our conditions seems, therefore, to call for some other explanation. We must look for some unifying idea that accounts for perception of a single unified object when the magnitude of disparity exceeds what is usual, or even normally possible, and also when much of the display lies outside, or is unrelated to, Panum's area.

We approach an explanation by noting that certain of the well-known constancies were not maintained under our experimental conditions, and by examining one aspect of three of these constancies; the frequency of their "not holding." The breakdown of veridicality is analyzed and shown in Table 1. It can be seen that the three categories, object unity, brightness unity, and form constancy, show different frequencies of breakdown, respectively 2,7 , and 12 times. Our explanation will propose a reason for these differences.

From a functional and molar theory of perceptual processes, an organism is designed (within its limitations) to perceive veridicality, which serves to preserve the individual organism and its species. When stimulus conditions exceed normal limits, and veridicality partially breaks down, as in this study, what "holds" 
and what does not "hold" is, therefore, of interest.

In normal binocular vision complete veridicality is present when none of the visual constancies fail and visual discriminations are optimal. The constancies and discriminatory abilities can be ranked in a hierarchy of survival value. Color constancy, for example, is not as necessary for human survival as brightness constancy and would rank lower. Motion detection would likely rank higher than visual acuity.

In this study, the excess of visual information given regarding the stimulus objects when binocular disparity was extranormal can be thought of as overloading the neural processes involved in the maintenance of object unity, brightness unity, and form constancy. One plausible ordering of these aspects of veridicality would place unity of the object first as a factor contributing to a continuing stability of the visual world. An organism with binocular vision has a better chance of survival with single rather than diplopic vision. Fleeing from or attacking the "wrong" object lowers probability of survival. Brightness unity (or constancy) would rank second. Loss of brightness constancy with changes in illumination or reflectance lowers the stability of the visual world. A natural example that takes advantage of this fact is the defensive camouflage used by various species where brightness constancy itself is exploited as a means of concealment. If, however, brightness were directly and absolutely linked to the stimulus energy input (i.e., there was no Weber-Fechner function) the visual world would likely appear as a kaleidoscopic hodge-podge much of the time. Form constancy would rank third. A single form with constant brightness might change shape as, for example, when a rabbit elongates and contracts when running, yet it remains recognizable to a predator as its prey.

From this discussion, the breakdown of veridicality should occur most often in form constancy, which is ranked least important; least often in object unity, which is ranked most important; and a middling number of times with brightness unity, which is ranked between the other two. The sums below the right side of Table 1 show this to be the case.
The hierarchy proposed offers a plausible explanation of the analysis of the results, but is, of course, an ad hoc idea. Support for this ad hoc explanation of the results will be forthcoming if a different family of stimulus objects yields a similar hierarchy of breakdowns in veridicality. Such an experiment is in progress.

\section{REFERENCES}

GIBSON, J. J. The perception of the visual world. Boston: Houghton Mifflin, 1950.

GROMAN, W. D. An apparatus for presenting continuously varying binocularly disparate views of an apparently single object. Quarterly Journal of Experimental Psy chology, 1964, 16, 184-186.

ITTELSON, W. H. Visual space perception. New York: Springer, 1960.

KOHLER, I. Experiments with goggles. Scientific American, 1962, 206 62-72.

LEIBOWITZ, H. W. Visual perception. New York: Macmillan, 1965

OGLE, K. N. Researches in binocular vision. Philadelphia: W. B. Saunders, 1950.

OSGOOD, C. E. Method and theory in experimental psychology. New York: Oxford University Press, 1953.

TREISMAN, A. Binocular rivalry and stereoscopic depth perception Quarterly Journal of Experimental Psychology, 1962, 14, 23-27.

\section{NOTES}

1. Address: Department of Psychology, Virginia Commonwealth University, 810 West Franklin Street, Commonwealth of Virginia, Richmond, Virginia 23220.

2. Exposure of the stimuli individually to over 150 students from psychology classes, ignorant of the fact that one of two identical stimulus objects was presented to each eye, resulted $100 \%$ of the time in a description of the perceived object as a perfectly normal apparently single object when viewed initially. After viewing the various changing phenomena, the nature of the stimulus conditions were shown to the students. Almost all showed marked surprise that two objects were involved. Neither the initial singularity nor the reality of the perceived object was ever questioned.

3. The verbal description here would seem to imply a contradiction. How can there be interpenetration and, at the same time, a striking solidity and unity of the perceived object? We can only state that the paradox is a semantic one. Perceptually, the unity and solidity of the figure is quite convincing.

(Accepted for publication January 20, 1969.) 